Supplementary Information for

\title{
Bioinspired Crowding Inhibits Explosive Ice Growth in Antifreeze Protein Solutions
}

Zhang Liu, ${ }^{1}$ Yan Wang, ${ }^{2}$ Xia Zheng,,${ }^{1,3}$ Shuo Liu, ${ }^{1}$ Shenglin Jin, ${ }^{1}$ Zhiyuan He, ${ }^{1}$ Jun-Feng Xiang, ${ }^{4,5}$ Jianjun Wang ${ }^{* 1,3,4}$

${ }^{1}$ Key Laboratory of Green Printing, Beijing National Laboratory for Molecular Science, Institute of Chemistry, Chinese Academy of Sciences, Beijing 100190, China

${ }^{2}$ School of Medicine, Shihezi University, Shihezi, Xinjiang 832000, China

${ }^{3}$ School of Future Technology, University of Chinese Academy of Sciences, Beijing 100049, China

${ }^{4}$ School of Chemical Sciences, University of Chinese Academy of Sciences, Beijing 100190, China

${ }^{5}$ CAS Research/Education Center for Excellence in Molecular Sciences, and Beijing National Laboratory for Molecular Science, Institute of Chemistry, Chinese Academy of Sciences, Beijing 100190, P. R. China

*Corresponding Author (s): Jianjun Wang: wangj220@iccas.ac.cn 


\section{The information about Type III AFP, MpdAFP, MpAFP and AFGP8.}

Type III AFP (PBD ID 3RDN), ${ }^{1}$ MpdAFP, ${ }^{2}$ and $M p$ AFP (PBD ID 3P4G) ${ }^{3}$ were expressed and purified as described in the corresponidng literatures. The protein sequences are shown in Figure S1a, and the purities are presented by SDSPAGE (Figure S1b). As the MW is a key factor of our mechanistic hypothesis. We further performed MALDI-TOF MS and conformed the exact MW of the three AFPs, as indicated in Figure S1c, the exact MW of Type III AFP, MpdAFP and $M p A F P$ are $8 \mathrm{kDa}, 24 \mathrm{kDa}$ and $32 \mathrm{kDa}$, respectively. The MpdAFP is a fusion protein with Thioredoxin, which is expressed and purified following the procedures described in references. ${ }^{2,4}$ The gene sequence of the fusion protein is shown in Figure S2.

(a)

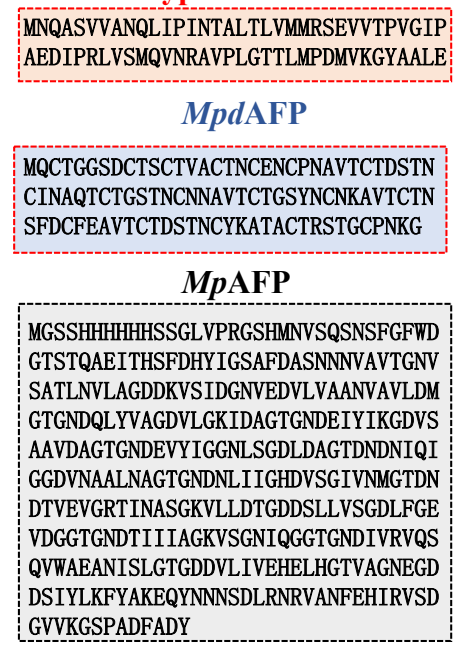

(b)

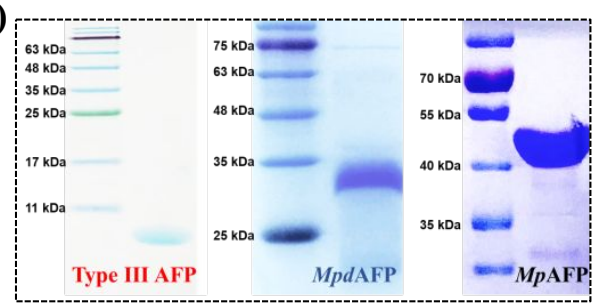

(c)

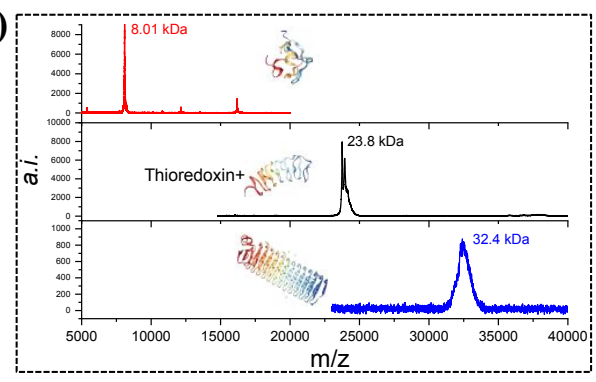

Figure S1. The protein sequences (a), the SDS-PAGE results (b) and the MALDI-TOF MS results (c) of Type III AFP, MpdAFP, and MpAFP. The his-tag (MGSSHHHHHHSSGLVPR[cleave site]GSHM) is cleaved at the thrombin cleavage site after purification.

(a)

ATGAGCGATAAAATTATTCACCTGACTGACGACAGTTTTGACACGGATGTA CTCAAAGCGGACGGGGCGATCCTCGTCGATTTCTGGGCAGAGTGGTGCGGT CCGTGCAAAATGATCGCCCCGATTCTGGATGAAATCGCTGACGAATATCAG GGCAAACTGACCGTTGCAAAACTGAACATCGATCAAAACCCTGGCACTGCG CCGAAATATGGCATCCGTGGTATCCCGACTCTGCTGCTGTTCAAAAACGGT GAAGTGGCGGCAACCAAAGTGGGTGCACTGTCTAAAGGTCAGTTGAAAGAG TTCCTCGACGCTAACCTGGCCGGTTCTGGTTCTGGCCATATGCACCATCAT CATCATCATTCTTCTGGTCTGGTGCCACGCGGTTCTGGTATGAAAGAAACC GCTGCTGCTAAATTCGAACGCCAGCACATGGACAGCCCAGATCTGGGTACC GACGACGACGACAAGGCCATGGCTGATATCGGATCCGAATTC ATGCAATGCACTGGAGGTTCTGATTGTACTAGTTGTACAGTAGCGTGCACC AATTGTGAAAACTGTCCAAATGCAGTAACGTGTACCGATTCTACAAATTGT ATAAATGCTCAAACCTGTACTGGGTCTACAAACTGCAATAATGCGGTGACC TGTACTGGGTCTTACAACTGCAATAAAGCGGTGACGTGTACGAACTCGTTC GACTGTTTTGAAGCCGTTACATGTACTGATTCGACCAACTGTTACAAAGCT ACAGCATGCACTCGTTCAACAGGATGTCCAAATAAAGGTTAA (b) Sequence coverage: $86.0 \%$
10
20
30
40
50
60 MSDKIIHLTD DSFDTDVLKA DGAILVDFWA EWCGPCKMIA PILDEIADEY QGKLTVAKLN \begin{tabular}{cccccr}
70 & 80 & 90 & 100 & 110 & 120 \\
IDQNPGTAPK & YGIRGIPTLL & LFKNGEVAAT & KVGALSKGQL & KEFLDANLAG SGSGHMHHHH \\
\hline
\end{tabular}

\begin{tabular}{cccccr}
130 & 140 & 150 & 160 & 170 & 180 \\
HHSSGLVPRG SGMKETAAAK & FERQHMDSPD & LGTDDDDKAM ADIGSGPMQC & TGGSDCTSCT \\
\hline & & & & & \\
190 & 200 & 210 & 220 & 230 & 240 \\
VACTNCENCP NAVTCTDSTN & CINAQTCTGS & NNCNNAVTCT & GSYNCNKAVT & CTNSFDCFEA \\
\hline
\end{tabular}

$\begin{array}{ccc}250 & 260 \\ \text { VTCTDSTNCY KATACTRSTG CPNKG } & \mathbf{M}_{\mathbf{W}}=\mathbf{2 3 . 7 8} \mathbf{~ k D a}\end{array}$

Figure S2. (a) The gene sequence of fusion protein of Thioredoxin (Red) with MpdAFP (Blue). (b) The expected (black and red) and detected (red) amino acid sequence of MpdAFP.

\section{The molecular structure, the result of HLPC, and MALDI-TOF MS of AFGP8.}



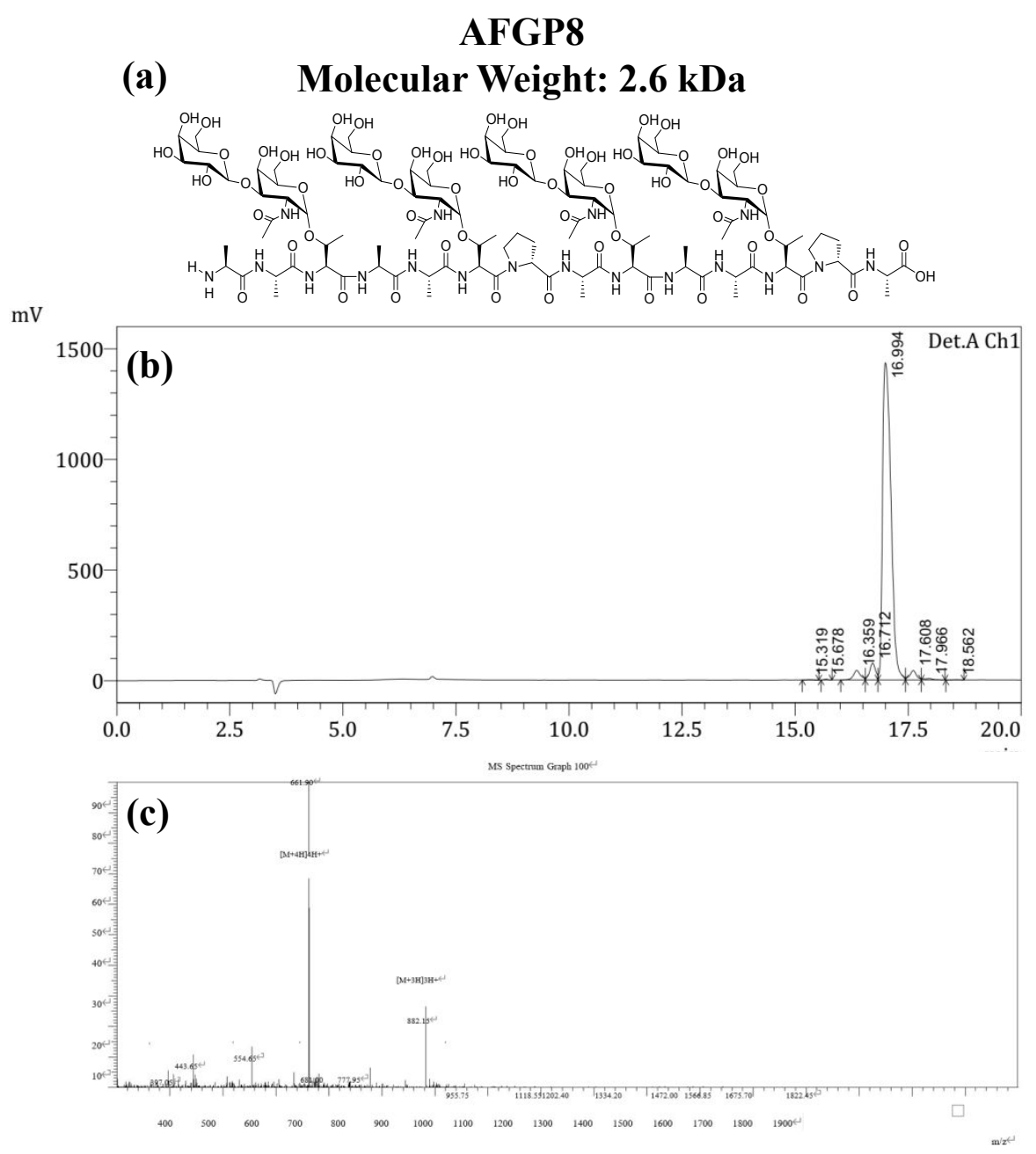

Figure S3. The molecular structure (a), result of HLPC (b) and MALDI-TOF MS (c) of AFGP8.

\section{Derive the phase boundaries of LLPS in the PEG/SC system.}

Liquid-liquid phase separation (LLPS) can be constructed by polyethylene glycol 6000 (PEG) and dehydration salt sodium citrate (SC) at certain concentrations and mixing ratios. The phase boundaries have been determined by turbidity changes in the mixing process of SC with PEG. Figure S4a gives a series of turbidity curves with a fixing PEG concentration and increasing SC concentration. Generally, all of the curves go through three regions. Before the addition of SC, the PEG solution is transparent and gives zero absorbencies. With the consecutive titration of SC into PEG solution under stirring, the turbidity remains close to zero until the concentration of SC reaches a critical value $C$, at which the turbidity increases sharply and small condensed droplets start to form in the solution. The turbidity keeps increasing with the continuous addition of SC and then reaches the plateau and keeps unchanged thereafter. The critical concentration $C$ is regarded as the region boundary between the homogenous PEG/SC mixture and the LLPS. The turbid suspension of amorphous droplets will coalesce into a top phase (C-LLPS) if stop stirring, leaving the dilute phase as a bottom phase (D-LLPS). From the turbidity curves, we can get the phase diagram in Figure S4b.

(a)

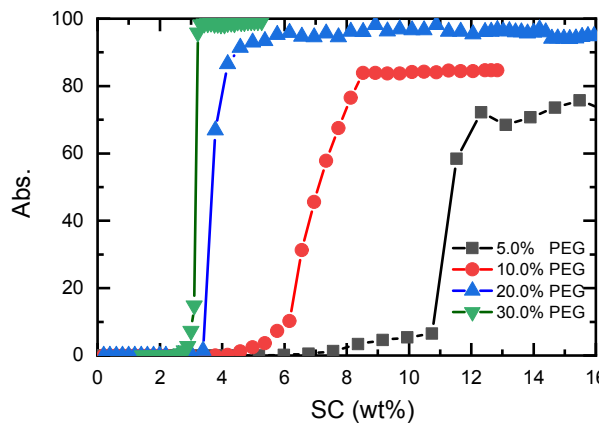

(b)

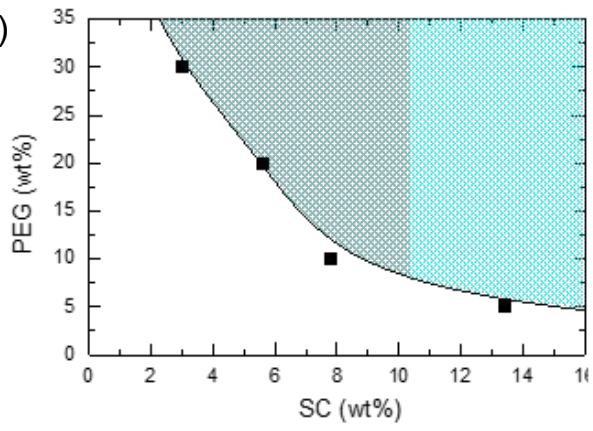


Figure S4. Turbidity against SC concentration at different PEG concentrations (a) and the LLPS phase boundaries of the PEG/SC system (b).

\section{The morphology of C-LLPS observed by Cryo-SEM}

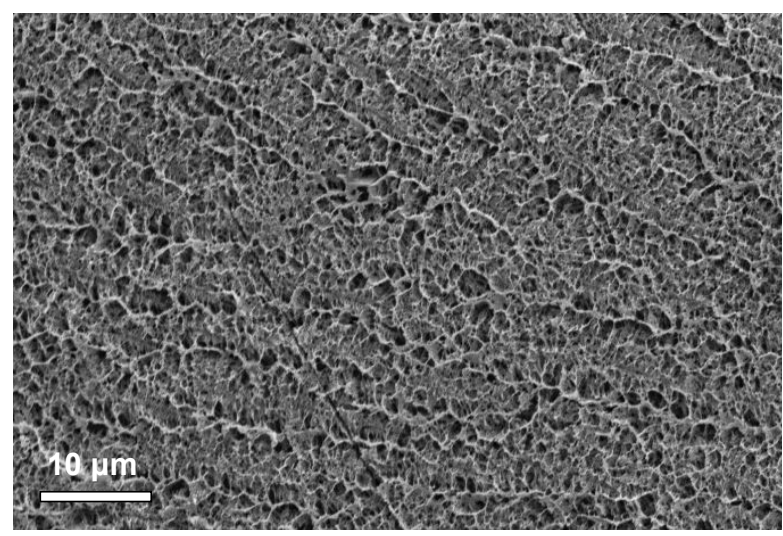

Figure S5. Cryo-SEM image of C-LLPS containing $19 \% \mathrm{PEG} / 7 \% \mathrm{SC}$.

\section{The mass concentrations of PEG ( $\left.\mathrm{W}_{\mathrm{PEG}}\right)$ and $\mathrm{SC}\left(\mathrm{W}_{\mathrm{SC}}\right)$ in the C-LLPS characterized by the refractive index method.}

The mass concentrations of SC in this series of C-LLPS was determined by flame photometry. ${ }^{[5]}$ The concentration of PEG in those C-LLPSs has been characterized by the refractive index method. ${ }^{[6]}$ Briefly, since the refractive index of the samples depends on the PEG and salt concentrations, calibration plots of refractive index versus polymer concentration were prepared for different concentrations of SC (Figure S6). The following equation describes the relation between the refractive index (n) and the PEG $\left(\mathrm{W}_{\mathrm{PEG}}\right)$ and $\mathrm{SC}$ ( $\mathrm{W}_{\mathrm{SC}}$ ) mass fractions: $\mathrm{n}=\mathrm{a}_{0}+\mathrm{a}_{1} \mathrm{~W}_{\mathrm{PEG}}+\mathrm{a}_{2} \mathrm{~W}_{\mathrm{SC}}$, where $\mathrm{a}_{0}=1.3325$ is the refractive index of pure water and $\mathrm{a}_{1}=1.48 \times 10^{-3}$ and $\mathrm{a}_{2}=1.79 \times 10^{-3}$ are derived from Figure $\mathrm{S} 6$.
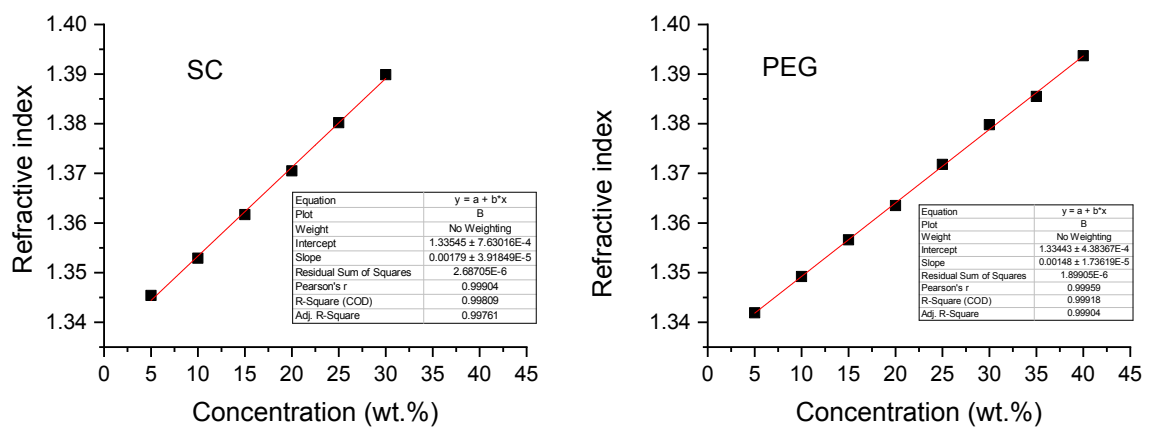

Figure S6. Calibration plots of refractive index versus PEG or SC concentration.

6. The inhibition of ice growth in C-LLPS solutions containing different kinds of AFPs.
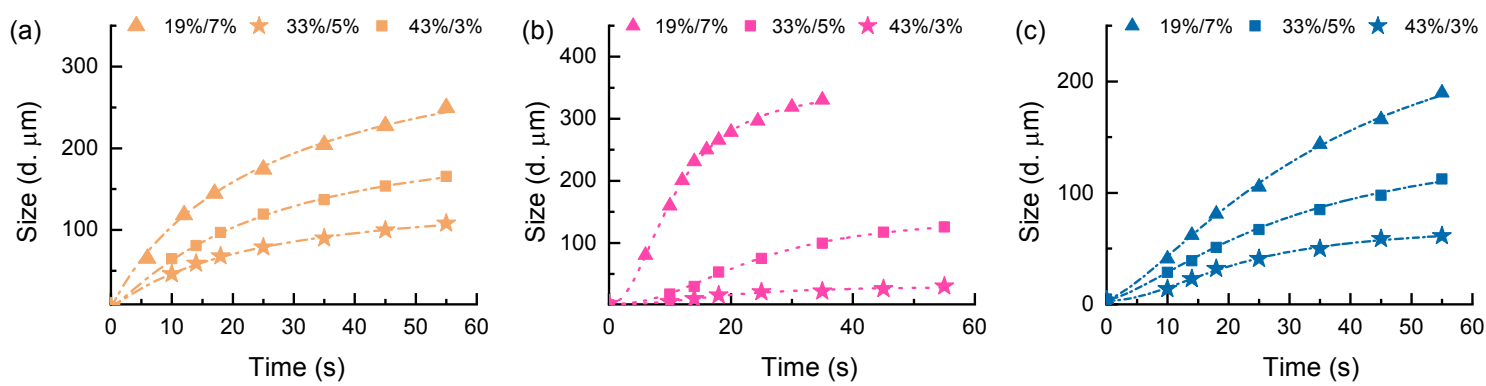

Figure S7. Change of ice crystal size with time in solutions of C-LLPSs with Type III AFP at $\Delta \mathrm{T}=\mathrm{TH}+$ 
$0.05^{\circ} \mathrm{C}$ (a), MpdAFP at $\Delta \mathrm{T}=1.55^{\circ} \mathrm{C}(\mathrm{b})$, and $\mathrm{MpAFP}$ at $\Delta \mathrm{T}=1.35^{\circ} \mathrm{C}$ (c). The concentration of AFPs are $0.50 \mathrm{mg} / \mathrm{mL}$ (Type III AFP), $0.20 \mathrm{mg} / \mathrm{mL}$ (MpdAFP) and $0.10 \mathrm{mg} / \mathrm{mL}$ (MpAFP) respectively.

7. The inhibition of explosive ice growth in C-LLPS/AFGP solution.

\section{(a) AFGP in PBS}
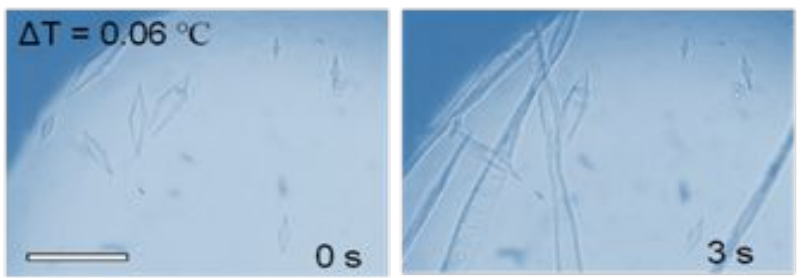

(b) AFGP in C-LLPS

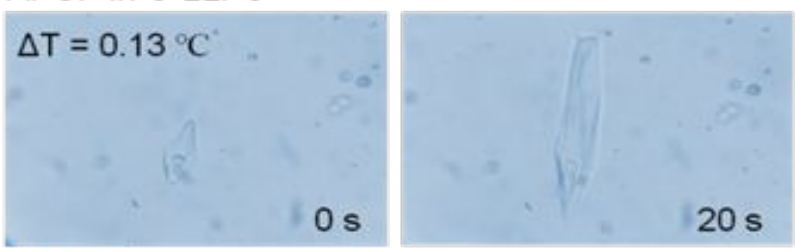

Figure S8. The ice growth process in solutios of $1.00 \mathrm{mg} / \mathrm{mL}$ AFGP8 when dissolved in PBS solutions (a) and in C-LLPS containing 33\% PEG/5\% SC (b). Scale bar $=50 \mu \mathrm{m}$.

8. The inhibition of explosive ice growth in C-LLPS/Type III AFP and C-LLPS/MpdAFP solution.
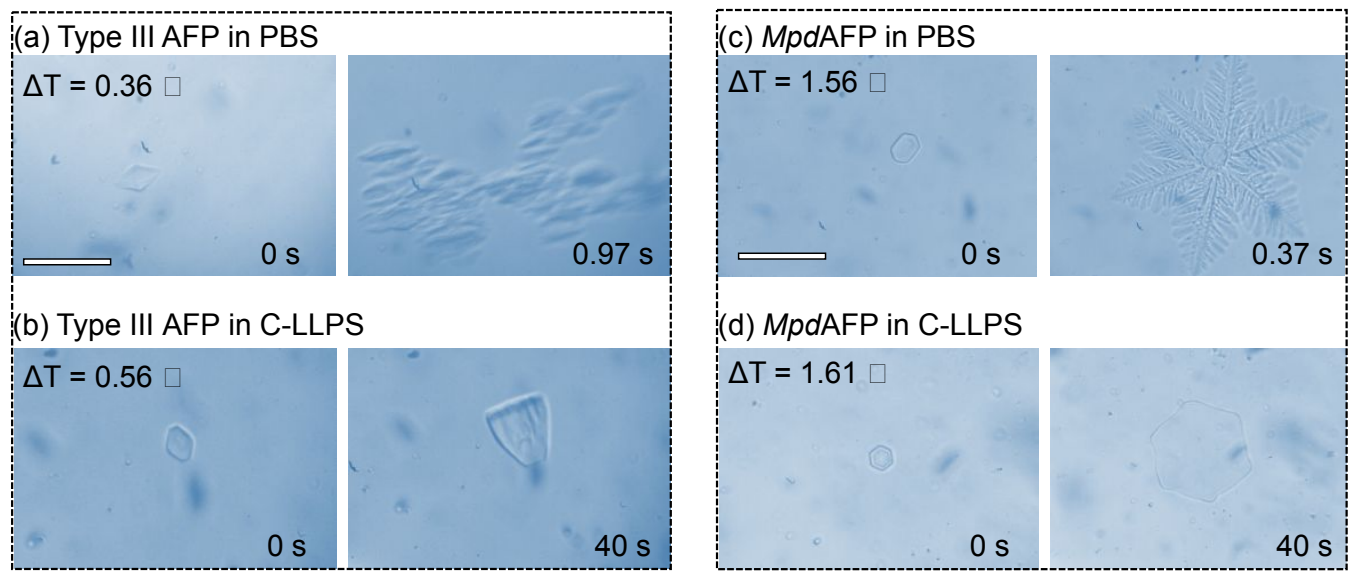

Figure S9. The ice growth process in solutions of $0.50 \mathrm{mg} / \mathrm{mL}$ Type III AFP when dissolved in PBS (a) and C-LLPS solution containing $43 \% \mathrm{PEG} / 3 \% \mathrm{SC}$ (b). The ice growth in solutions of when $0.20 \mathrm{mg} / \mathrm{mL} \mathrm{MpdAFP}$ when dissolved in PBS (c) or in C-LLPS solution containing 33\% PEG/5\% SC (d). Scale bar $=100 \mu \mathrm{m}$.

\section{The TH changes of MpdAFP in C-LLPSs with increasing crowdedness.}




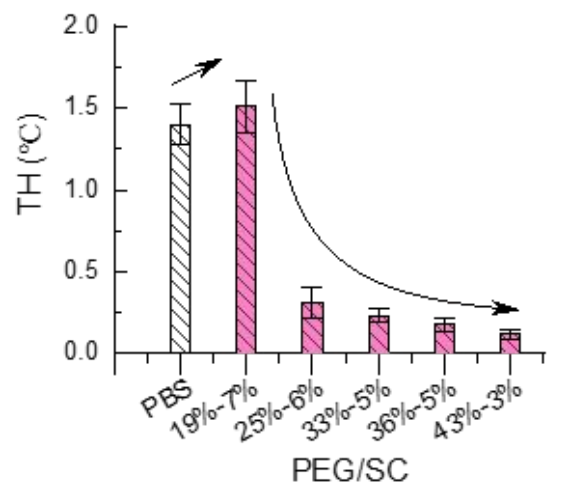

Figure S10. The TH changes of $0.20 \mathrm{mg} / \mathrm{mL}$ MpdAFP with increasing crowdedness of C-LLPSs.

\section{The hydrogen bonds formed between $\mathrm{H}_{2} \mathrm{O}$, PEG and SC.}

NOE effect is a powerful tool to probe the spatial relationship between atoms or groups within a molecule or between different molecules. In the present work, the 1D selective gradient NOE was used to reveal the possible hydrogen bonds formed between each component in the C-LLPS solutions. The proton assignments and ${ }^{1} \mathrm{H}$ NMR spectra of $\mathrm{H}_{2} \mathrm{O}$ (s, $5.62 \mathrm{ppm}, 273.2 \mathrm{~K}$ ), $\mathrm{PEG}$ (t, $4.32 \mathrm{ppm}, 273.2 \mathrm{~K}$ ) and $\mathrm{SC}$ (d, $3.26 \mathrm{ppm}, 273.2$ $\mathrm{K})$ are shown in Figure S11a. In the 1D selective gradient NOE experiments, when a specific proton is excited, only protons within $5 \AA$ can give a signal response. ${ }^{[7]}$ As shown in Figure S11b, S11c and S11d, it has been found when one of the three components was excited, the resonance at the position of the other two components can be observed. Therefore, it can be concluded that the $\mathrm{H}_{2} \mathrm{O}$, PEG, and SC molecules are spatially very closed to each other, i.e. within a distance less than $5 \AA$. Taking account of the strong hydrogen bonds forming ability of the EG groups and the SC molecules, it is reasonable to conclude that hydrogen bonds are forming among the $\mathrm{H}_{2} \mathrm{O}$, PEG and SC molecules. Meanwhile, the chemical shift of the protons on the water molecules moves to upfield significantly when the temperature increases from $298.2 \mathrm{~K}$ to $338.2 \mathrm{~K}$, as shown in Figure S12, which strongly supports the formation of an extended hydrogen bond at room temperature $(298.2 \mathrm{~K})$ and the breaking of this hydrogen networks at a higher temperature in the crowded solutions.
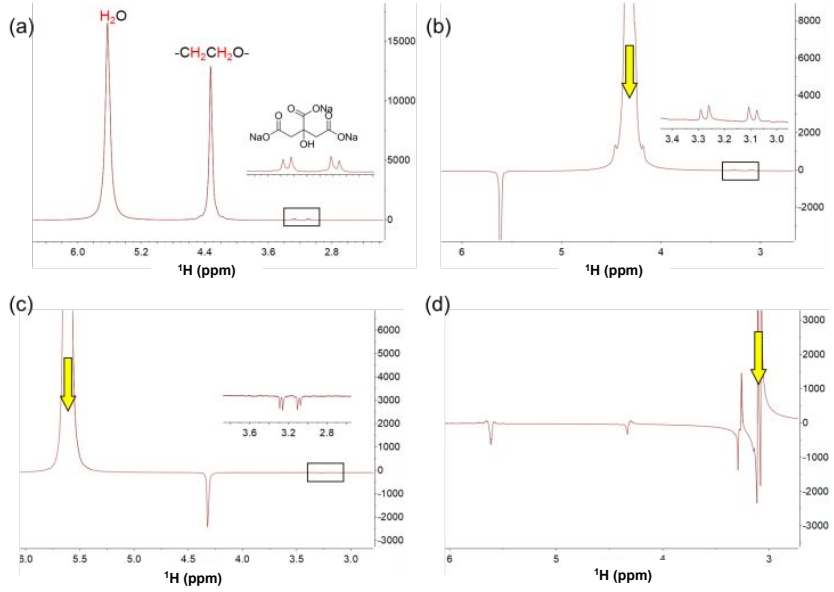

Figure S11. The ${ }^{1} \mathrm{H}-\mathrm{NMR}$ (a) and 1D selective gradient NOESY spectrum of crowded solutions containing $33 \% \mathrm{PEG} / 5 \% \mathrm{SC}$, when the protons on PEG (b), $\mathrm{H}_{2} \mathrm{O}$ (c), and SC (d) were selected to be excited (yellow arrows). The experiments were performed at $273.2 \mathrm{~K}$. 


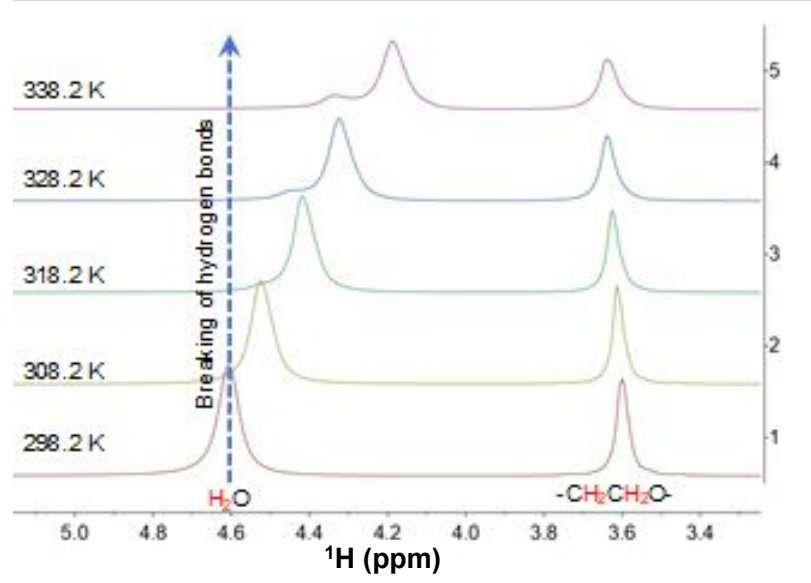

Figure S12. The upfield shift of the protons on $\mathrm{H}_{2} \mathrm{O}$ in the crowded solutions containing $33 \% \mathrm{PEG} / 5 \% \mathrm{SC}$, when the testing temperature increases from $298.2 \mathrm{~K}$ to $338.2 \mathrm{~K}$, indicates the breaking of the hydrogen bonds.

\section{The DSC of EG, PEG and C-LLPS solutions of different concentrations.}

Both PEG and C-LLPS show obvious capability of water confinement. The C-LLPSs is much more effective than that of the pure PEG solutions in confining water, as the fraction of $\mathrm{CW}$ among all freezable water increases to almost $100 \%$ in the C-LLPS containing $43 \% \mathrm{PEG} / 3 \% \mathrm{SC}$ while that of corresponding pure PEG solutions is only around $15 \%$ to around $20 \%$ as shown in Figure S13.

(a)

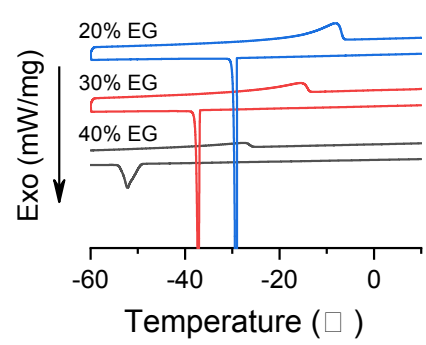

(b)

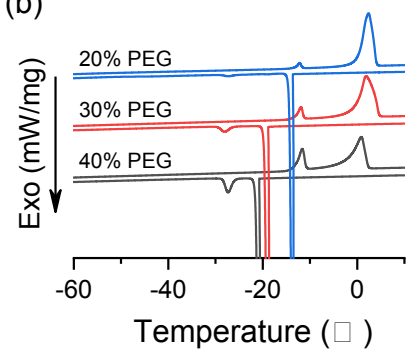

(c)

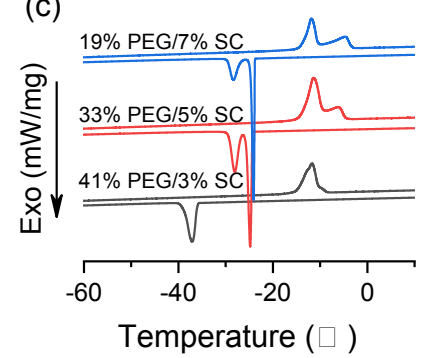

Figure S13. DSC freezing/heating traces of EG (a), PEG (a) and C-LLPS (c) with increasing concentration.

\section{References:}

[1] Jia, Z.; DeLuca, C. I.; Chao, H.; Davies, P. L., Structural Basis for the Binding of a Globular Antifreeze Protein to Ice. Nature 1996, 384, 285-288..

[2] Qiu, L. M.; Ma, J.; Wang, J.; Zhang, F. C.; Wang, Y., Thermal Stability Properties of an Antifreeze Protein from the Desert Beetle Microdera Punctipennis. Cryobiology 2010, 60, 192-197.

[3] Garnham, Christopher P.; Gilbert, Jack A.; Hartman, Christopher P.; Campbell, Robert L.; LaybournParry, J.; Davies, Peter L., A Ca ${ }^{2+}$-Dependent Bacterial Antifreeze Protein Domain Has a Novel B-Helical Ice-Binding Fold. Biochem. J 2008, 411, 171-180.

[4] Mao, X.; Liu, Z.; Ma, J.; Pang, H.; Zhang, F., Characterization of a Novel B-Helix Antifreeze Protein from the Desert Beetle Anatolica Polita. Cryobiology 2011, 62, 91-99.

[5] Zafarani-Moattar, M. T.; Sadeghi, R.; Hamidi, A. A., Liquid-Liquid Equilibria of an Aqueous TwoPhase System Containing Polyethylene Glycol and Sodium Citrate: Experiment and Correlation. Fluid Phase Equilib. 2004, 219, 149-155.

[6] Cheluget, E. L.; Gelinas, S.; Vera, J. H.; Weber, M. E., Liquid-Liquid Equilibrium of Aqueous Mixtures 
of Poly(Propylene Glycol) with Sodium Chloride. J. Chem. Eng. Data 1994, 39, 127-130.

[7] Kessler, H., Gehrke, M., Griesinger, C., Two-Dimensional NMR Spectroscopy: Background and

Overview of the Experiments [New Analytical Methods (36)]. Angew. Chem., Int. Ed. 1988, 27, 490-536. 